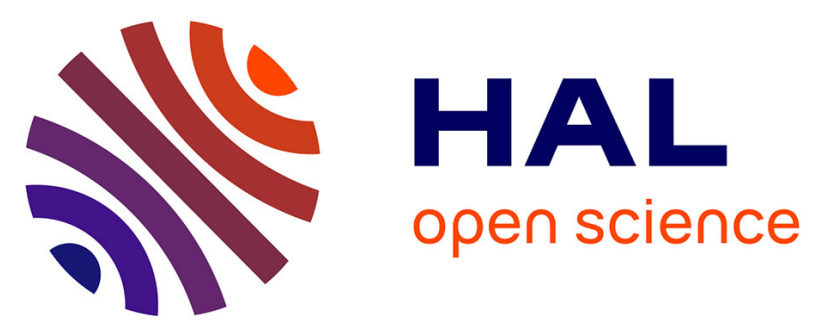

\title{
Effects of polarization mode dispersion on an all polarization maintaining fibers based coupled optoelectronic oscillator
}

Alexis Bougaud, Aliou Ly, Gilles Bailly, Arnaud Fernandez, Olivier Llopis

\section{- To cite this version:}

Alexis Bougaud, Aliou Ly, Gilles Bailly, Arnaud Fernandez, Olivier Llopis. Effects of polarization mode dispersion on an all polarization maintaining fibers based coupled optoelectronic oscillator. IFCSISAF 2020, IEEE International Frequency Control Symposium and IEEE International Symposium on Application of Ferroelectrics joint conference, Jul 2020, Keystone (virtual conference), United States. 10.1109/IFCS-ISAF41089.2020.9234855 . hal-02975556

\author{
HAL Id: hal-02975556 \\ https://hal.laas.fr/hal-02975556
}

Submitted on 22 Oct 2020

HAL is a multi-disciplinary open access archive for the deposit and dissemination of scientific research documents, whether they are published or not. The documents may come from teaching and research institutions in France or abroad, or from public or private research centers.
L'archive ouverte pluridisciplinaire HAL, est destinée au dépôt et à la diffusion de documents scientifiques de niveau recherche, publiés ou non, émanant des établissements d'enseignement et de recherche français ou étrangers, des laboratoires publics ou privés. 


\title{
Effects of polarization mode dispersion on an all polarization maintaining fibers based coupled optoelectronic oscillator
}

\author{
Alexis Bougaud, Aliou Ly*, Gilles Bailly, Arnaud Fernandez, Olivier Llopis \\ LAAS-CNRS, Université de Toulouse, CNRS, UPS, Toulouse, France \\ *A. Ly is today with Laser Quantum Ltd, Stockport, UK \\ Contact author: abougaud@laas.fr
}

\begin{abstract}
We report the optimization of a $30 \mathrm{GHz}$ COEO by using an all polarization maintaining fiber (PMF) to design its optical loop. We unexpectedly found that polarization mode dispersion (PMD) can have detrimental effects on the COEO performances. Indeed, with a $400 \mathrm{~m}$ SMF fiber spool in the optical cavity, a phase noise level as low as $-115 \mathrm{dBc} / \mathrm{Hz}$ at $1 \mathrm{kHz}$ offset frequency was obtained. Conversely, with a PMF fiber spool, only a phase noise level as low as $-100 \mathrm{dBc} / \mathrm{Hz}$ and $-70 \mathrm{dBc} / \mathrm{Hz}$ at $1 \mathrm{kHz}$ offset frequency for a $200 \mathrm{~m}$ PMF and $400 \mathrm{~m}$ PMF could be obtained respectively. PMD sets therefore an upper limit on the length of the PMF fiber that could be used.
\end{abstract}

Index Terms - Polarization mode dispersion; COEO; mode locked laser microwave photonics; polarization maintaining fiber; millimeter wave; phase noise.

\section{INTRODUCTION}

Optical approaches have seamlessly paved the way to the synthesis of high frequency (millimeter-wave) and spectrally pure RF signals which were beneficial to numerous applications these last decades [1]. The coupled optoelectronic oscillator (COEO) is among these solutions. As to mention, it has allowed the generation of electrical signals at $10 \mathrm{GHz}, 30 \mathrm{GHz}$ and $90 \mathrm{GHz}$ with phase noise levels at $1 \mathrm{kHz}$ offset frequency as low as $-120 \mathrm{dBc} / \mathrm{Hz}, \quad-114.4 \mathrm{dBc} / \mathrm{Hz}$ and $-104 \mathrm{dBc} / \mathrm{Hz}$ respectively [2,3]. A typical architecture of a COEO is shown in Fig. 1. It is a hybrid device composed of two coupled loops: an optical loop (black) and an opto-electrical loop (black and purple). The optical loop brings the required light energy necessary to the microwave oscillation of the optoelectronic loop through the semiconductor optical amplifier (SOA). A bandpass filter (CFBG) is also usually inserted inside the optical loop in order to limit the effect of the ASE to the noise of the COEO and to improve its overall stability.

The aforementioned results were obtained thanks to, among others, the fact that operation at any high frequency is theoretically possible [4] provided that one uses state-of-art optoelectronic components. This however might not be a cost effective solution to improve the spectral purity of RF signals generated from a COEO. Indeed, it seems more straightforward to optimize the design of the optical loop by carefully managing its intracavity dispersion [5] and/or by making it more compact [6].

Thus an all polarization maintaining fibers (PMFs) based COEO would be an interesting alternative. Indeed, in such a situation, a polarization controller (Fig. 1) is no longer needed.
However, we unexpectedly found that polarization mode dispersion (PMD) can have detrimental effects on a fully PMFs based COEO performances. The present paper aims at illustrating as well as discussing those unwanted effects and how can one circumvent them.

\section{OPTICAL FREQUENCY COMB OF A COEO WITH PMF AND SMF FIBERS AS AN OPTICAL DELAY LINE}

The COEO we want to optimize operates at $30 \mathrm{GHz}$ and its architecture is depicted in Fig. 1.

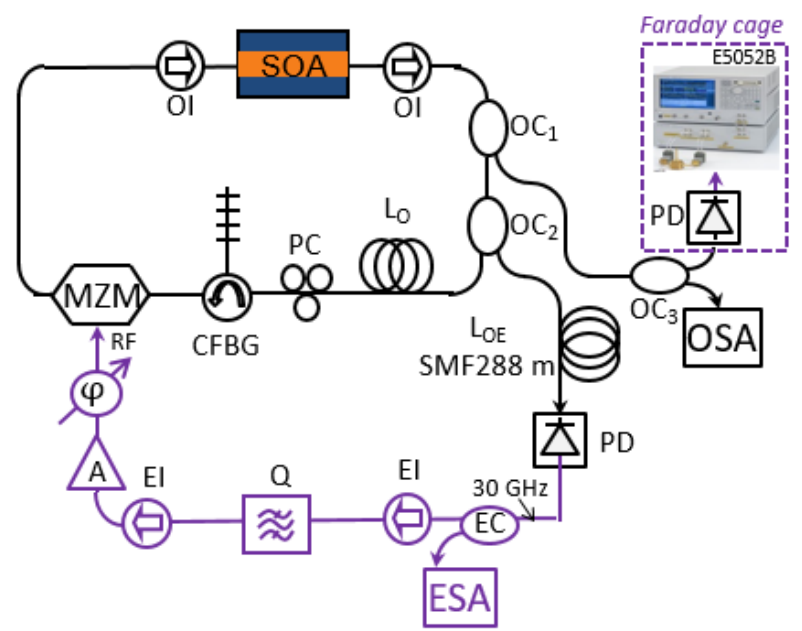

Fig. 1: Schematic of a $30 \mathrm{GHz}$ COEO. MZM: Mach Zehnder Modulator, SOA: Semiconductor Optical Amplifier, PC: Polarization Controller, ESA: Electrical Spectrum Analyzer. OSA: Optical Spectrum Analyzer. E5052B: phase noise analyzer. Lo: optical delay line. LoE: optoelectronic delay line. SMF: Single Mode Fiber. CFBG: Chirped Fiber Bragg Grating. OI: Optical Isolator. EI: Electrical Isolator. OC: Optical Coupler. EC: Electrical Coupler. A: RF amplifier.

It is the same as the one used in [3] where the optical delay line $\mathrm{L}_{\mathrm{O}}$ is made of $400 \mathrm{~m}$ SMF and more details about the other components can be found is this reference. The optimization consists simply in replacing the polarization controller and the optical delay line by a $400 \mathrm{~m}$ PMF fiber spool and see how the COEO performs. So we did and Fig. 2 compares the optical frequency comb of the COEO in two cases: with $400 \mathrm{~m} \mathrm{SMF}$ (Fig. 2(a)) and with $400 \mathrm{~m}$ PMF (Fig. 2(b)). 
We started with an observation of the optical spectrum since it reveals the phase matching quality between all modes that can oscillate. A wide span frequency comb with intense lines will result in ultra-short and low jitter optical pulses [7, 8] and thus in a low phase noise level for the corresponding RF signal accordingly. As it can be seen, when the optical delay line is made of a SMF fiber spool, the optical spectrum features intense lines which are nicely resolved, at least, with a precision set by the optical spectrum analyzer resolution $(0.08 \mathrm{~nm})$. Conversely, when a PMF fiber spool is used, significant differences appear. Firstly, with the $400 \mathrm{~m}$ PMF only (the PC in Fig. 1 is removed), there is no phase matching at all and the COEO is not modelocked. Secondly, with the presence of the PC that is rotated, a comb could be seen. However, it is less developed and less sharp than the comb in Fig. 2(a). Moreover, the phase matching does not cover the entire bandwidth of the chirped fiber Bragg grating. Undoubtedly, one should not expect to get a low phase noise COEO with such a spectrum. Last but not least, a red shift of both spectra is visible but it is more striking when PMF fibers are used. This red shift comes from the SOA [9] and it can be controlled by a proper dispersion management of the optical loop [5]. Thus, one can forebode that dispersive properties of the PMF fiber spool and of the CFBG, somehow might play an important role in the preceding results.

We therefore investigate in the next section, the dispersion properties of our PMF fiber spool and our CFBG.
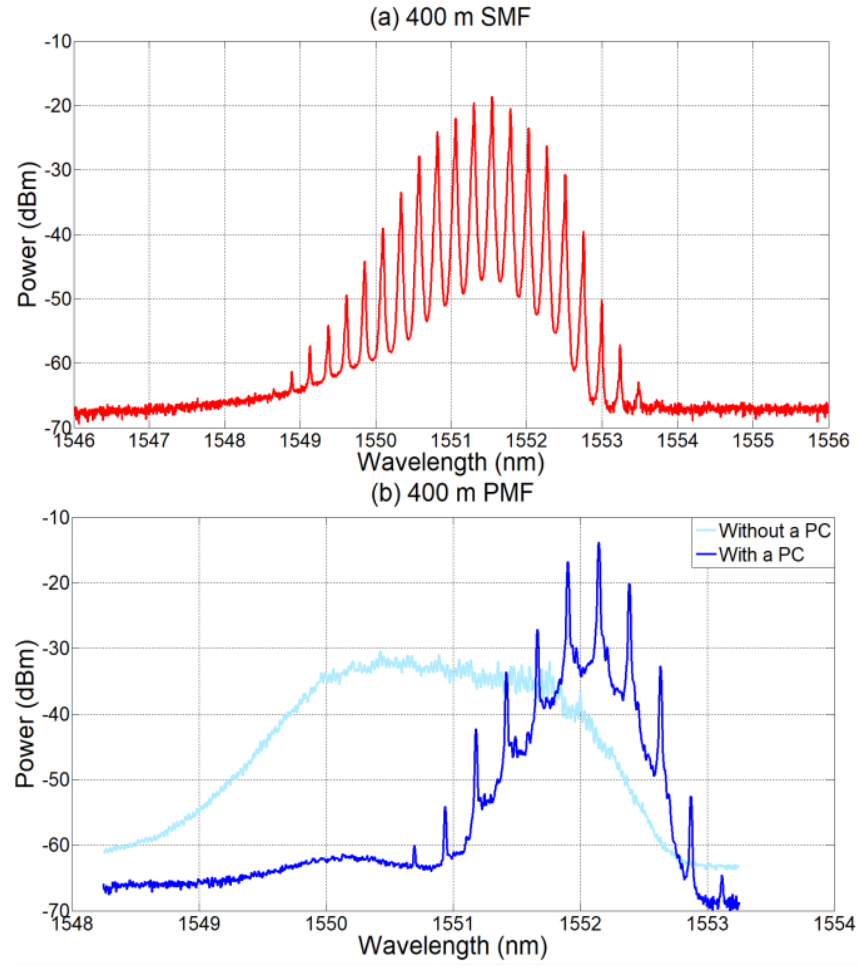

Fig. 2: Optical frequency comb of the $30 \mathrm{GHz}$ COEO in two cases. (a): with a $400 \mathrm{~m}$ SMF fiber spool as in Fig. 1. (b): with a $400 \mathrm{~m}$ PMF fiber spool with and without an insertion of a polarization controller.

\section{CHROMATIC AND POLARIZATION MODE DISPERSION MEASUREMENT}

We have recently introduced a simple and practical technique to measure the chromatic dispersion (CD) of optical fibers with lengths as short as a few hundred of meters for applications to a COEO [10]. Fig. 3 recalls this technique.

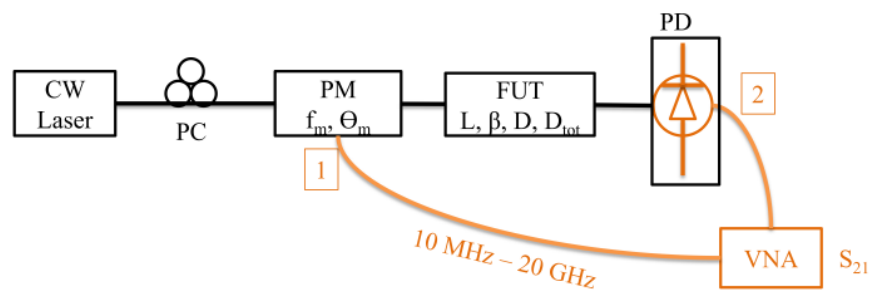

Fig. 3: Experimental setup for $\mathrm{CD}$ measurement: PC: polarization controller, PM: phase modulator, FUT: fiber under test, PD: photodiode, VNA: vector network analyzer, L: geometrical length, $\beta$ : complex propagation constant, D: CD per unit length of fiber in (ps/nm/km), $D_{\text {tot: }}$ accumulated CD over the length $\mathrm{L}$ in (ps/nm), $f_{\mathrm{m}}$ : RF signal frequency from $10 \mathrm{MHz}$ to $20 \mathrm{GHz}, \theta_{\mathrm{m}}$ : modulation depth.

It exploits the fact that the dispersion of the FUT will disturb the phase relationship between the two sidebands of the continuous-wave $(\mathrm{CW})$ phase modulated light. As a result, the gain of the $S_{21}$ parameter will be nonzero and from its measurement one can extract the CD as shown in Eq. (1).

$$
\begin{aligned}
& \left|S_{21}\right| \\
& =20 \log _{10}\left(\frac{\alpha \eta R_{L} P_{o p t} J_{0}\left(\theta_{m}\right) J_{1}\left(\theta_{m}\right) \sin \left(\frac{\pi L D}{c} \lambda^{2} f_{m}^{2}\right)}{\sqrt{R_{L}}}\right) \\
& -P_{R F}
\end{aligned}
$$

With $\alpha$ the FUT attenuation, $\eta$ the response of the photodiode in $A / W$ and $R_{L}$ the $50 \Omega$ load resistance of the VNA. Details of the extraction of $D_{\text {tot }}$ from this equation can be found in [10]. What is important is that a simple examination of the $S_{21}$ gain will provide enough information about how dispersive is the FUT as we will see.

\section{A. Dispersion of PMF and SMF fiber spools}

We measured and compared the dispersion of a $400 \mathrm{~m} \mathrm{SMF}$ fiber spool and of a $200 \mathrm{~m}$ PMF fiber spool. The CW laser operates at $1552 \mathrm{~nm}$ with an output power $\mathrm{P}_{\mathrm{opt}}$ of $13 \mathrm{dBm}$ and the RF power from the VNA $P_{R F}$ was fixed at $7 \mathrm{dBm}$. Fig. 4 displays the results obtained. For the $400 \mathrm{~m}$ SMF fiber spool, the $S_{21}$ amplitude evolves as expected. However, for the $200 \mathrm{~m}$ PMF fiber spool, oscillations are observed. In case of a standard fiber, these oscillations onset only for lengths of the order of hundreds of kilometers and the first zero is usually exploited to calculate the CD [11]. Thus, given the length of the PMF fiber spool, these oscillations come from another dynamic which is nothing but PMD that limits PMFs fibers performances [12]. Indeed in Fig. 4(b) we clearly see that with an insertion of a linear polarizer between the phase modulator and the FUT, one recovers the expected evolution for the $S_{21}$ gain. It is worth keeping in mind those results in Fig. 4(a) are obtained when the 
experimental setup is exactly as in Fig. 3. The linear polarizer is a PM slow axis aligned, thus it filters the polarization state of the incoming light along the PMF slow axis.

(a) Without an inline polarizer

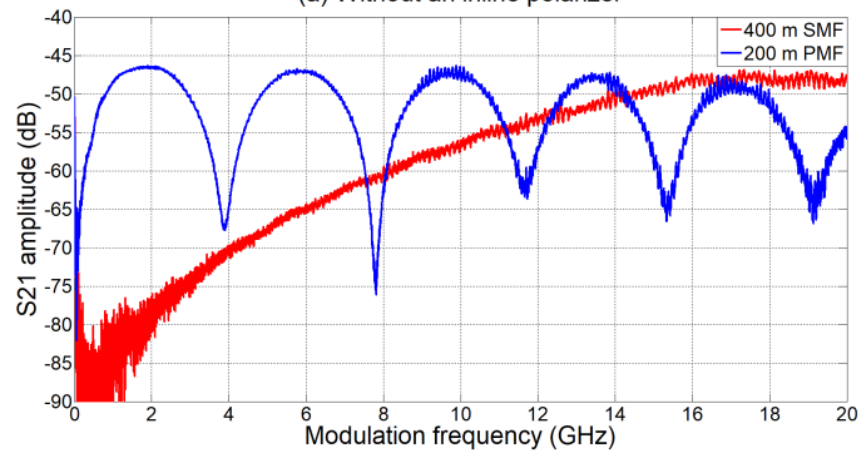

(b) With an inline polarizer

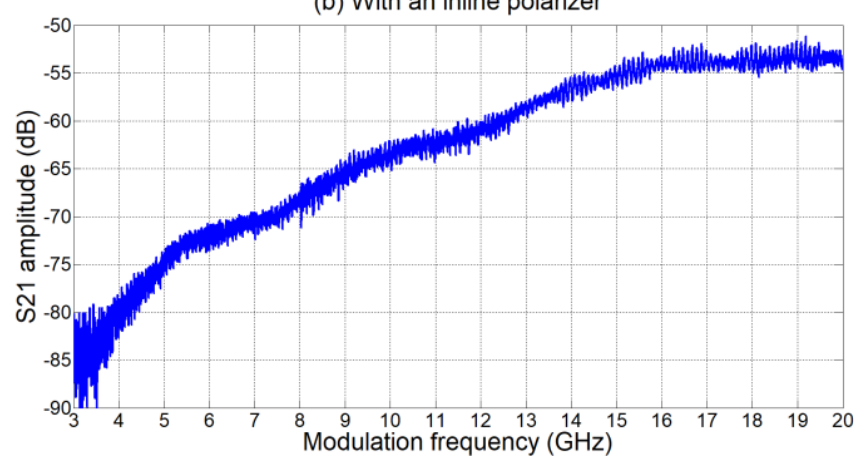

Fig. 4: Evolution of the $S_{21}$ gain for the two fiber spools studied in experimental conditions shown in Fig. 3.

\section{B. Transfer function and dispersion of the chirped fiber Bragg grating}

The method in Fig. 3 can also be used to fully characterize an optical bandpass filter like our CFBG which is used in reflection. Indeed, one needs just to have a tunable $\mathrm{CW}$ laser.

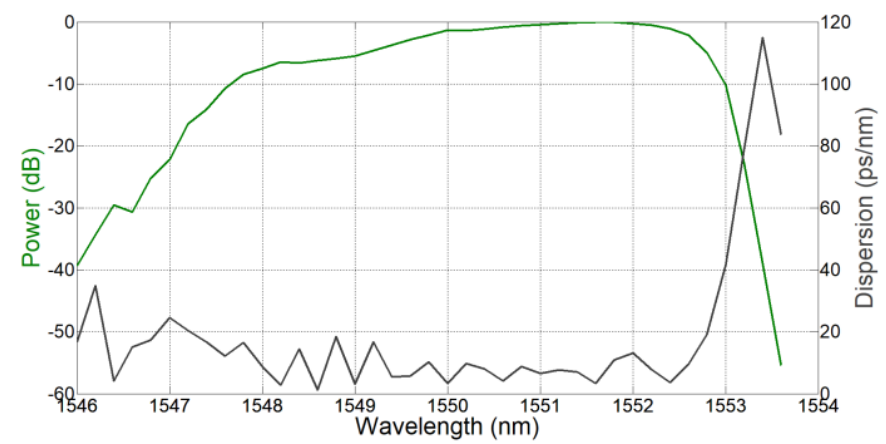

Fig. 5: Optical characteristics of our chirped fiber Bragg grating. Green: normalized reflected power. Dark gray: dispersion of the filter.

The dispersion is measured using Eq. (1) for different wavelengths and the transfer function is recovered by measuring the DC current at the photodiode output of Fig. 3 . The results are plotted in Fig. 5. The wavelengths range over which we could perform measurements was limited by the weak level of the DC current because of the high rejection of the CFBG. Under the bandwidth of the CFBG, the dispersion has an average value of $7 \mathrm{ps} / \mathrm{nm}$ that is in accordance with the $-7 \mathrm{ps} / \mathrm{nm}$ given by our provider. Indeed, the CFBG has two functions: it firstly limits the influence of the ASE and it secondly allows a management of the intracavity dispersion of the optical loop. However, close to the CFBG edges, the dispersion diverges up to more than $100 \mathrm{ps} / \mathrm{nm}$ but this does not have major impact on the COEO since the filter rejection is higher than $50 \mathrm{~dB}$.

Now let's see how one could optimize the COEO by taking into account these results.

\section{PERFORMANCES OF A FULL PMF FIBER BASED COEO}

We compared the performances in terms of optical frequency comb and spectral purity of a $30 \mathrm{GHz}$ COEO within two cases. In the former, the COEO is exactly as in Fig. 1. In the latter, the polarization controller is removed and an inline polarizer (the same as that one used for the results in Fig. 4(b)) is placed between the PMF fiber spool $(200 \mathrm{~m}$ or $400 \mathrm{~m}$ ) and the intensity modulator.

(a)

Optical spectrum

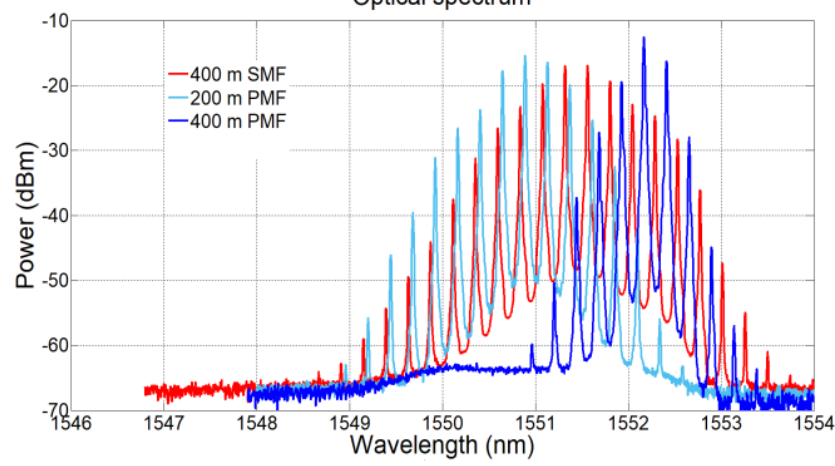

(b)

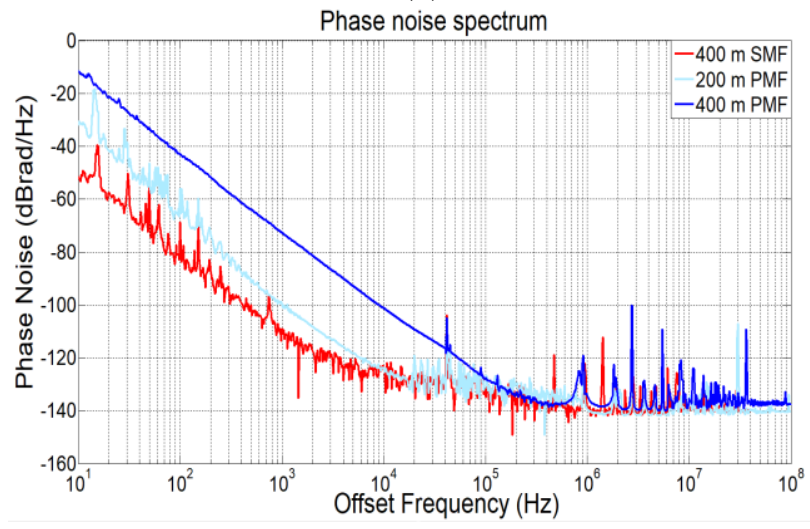

Fig. 6: Performances of the $30 \mathrm{GHz}$ COEO. (a): Optical spectrum. (b) Phase noise spectrum.

Overall, with the PMF fibers, the phase noise of the $30 \mathrm{GHz}$ COEO can never be better than the one obtained with the SMF fiber spool and the polarization controller (Fig. 6(b)). Moreover, when PMF fibers are used, the best results are obtained with a $200 \mathrm{~m}$ PMF fiber spool rather than with a $400 \mathrm{~m}$ PMF fiber spool. This is not only surprising but unexpected. Indeed, the quality factor provided by an optical delay line depends linearly with the delay it introduces and it is known 
that a high quality factor results in a good spectral purity of the RF carrier. An examination of the optical spectrum in Fig. 6(a) however shows that the spectrum obtained with a $400 \mathrm{~m}$ PMF fiber spool is not fully developed.

These salient differences are due the fact that PMD is a dynamical process that does exist in a PM fiber even if the input polarization is aligned properly along one of its Eigen axis [12]. Indeed, due to environmental mechanical fluctuations, a part of the light that is initially launched on one Eigen polarization of the PMF fiber, say the slow axis, will be coupled to the other Eigen polarization, say the fast axis, after a certain propagation distance. The fast axis optical power will then acquire randomly a part of the power of the slow one and create amplitude fluctuations in the loop. We therefore wished to estimate this effect by measuring the extinction ratio between the fast and slow axis of the $200 \mathrm{~m}$ and $400 \mathrm{~m}$ PM fiber using a Thorlabs ERM100 extinction ratio meter.

The results for a 5 min experiment are depicted in Figure 7. These curves highlight the chaotic coupling behavior between the fast and slow axis. The average extinction ratio is in agreement with the usual PMF specifications (around $25 \mathrm{~dB}$ ) but the value can drop down to $15 \mathrm{~dB}$ in the case of the $400 \mathrm{~m}$ spool (actually, two $200 \mathrm{~m}$ spools connected together).

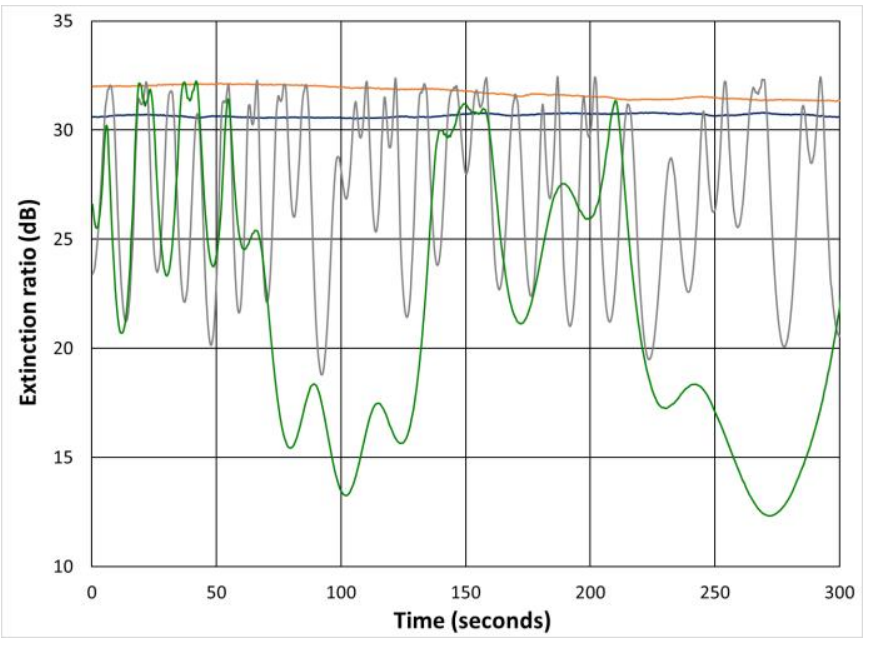

Fig. 7: Evolution versus time of the extinction ratio coefficient Er at the output of a PM fiber link with different fiber lengths. Blue curve: laser + fiber polarizer directly connected to the Er meter. Orange curve: same configuration with $1 \mathrm{~m}$ PM fiber added. Grey curve: one spool of $200 \mathrm{~m}$ PM fiber added. Green curve: $400 \mathrm{~m}$ PM fiber added (two $200 \mathrm{~m}$ spools).

The amplitude fluctuation brought by the Polarization Mode Coupling also induces a Differential Group Delay (DGD) that corresponds to the maximum difference in the propagation time of these two orthogonal modes due to PMD. This can thus bring an additional pulse to pulse timing jitter and enhance the RF phase noise.

\section{CONCLUSION}

We have, to the best of our knowledge, carried out for the first time a study of the performances of an all PMFs fibers based $\mathrm{COEO}$ in terms of phase noise level and optical frequency comb span. Taking advantage of a technique we developed recently, the existence of PMD in a PMF fiber spool was revealed. We have shown that by using a linear polarizer, the influence of PMD can be minimized, however, up to a certain extent. Indeed, even if doing so, PMD can reduce dramatically the system performance for long fiber delay.

\section{ACKNOWLEDGMENTS}

This work has been supported by the French national center for space studies (CNES) and the region Occitanie.

\section{REFERENCES}

[1] Quinlan, F., Ozharar, S., Gee, S., Delfyett, P. J. (2009). Harmonically modelocked semiconductor-based lasers as high repetition rate ultralow noise pulse train and optical frequency comb sources. Journal of Optics A: Pure and Applied Optics, 11(10), 103001.

[2] Yao, X. Steve, Larry Davis, and Lute Maleki. "Coupled optoelectronic oscillators for generating both RF signal and optical pulses." Journal of Lightwave Technology, vol. 18, ${ }^{\circ} 1$ (2000), pp. 73-78.

[3] Ly, A., Auroux, V., Khayatzadeh, R., Gutierrez, N., Fernandez, A. and Llopis, O. (2018). Highly spectrally pure $90 \mathrm{GHz}$ signal synthesis using a coupled optoelectronic oscillator. IEEE Photonics Tech. Letters, Vol. 30, July 2018, pp. 1313-1316.

[4] X.S. Yao, D. Eliyahu, L. Maleki, "Progress in the optoelectronic oscillatora ten year anniversary review," IEEE Microwave Theory and Tech. Symp. Digest, June 2004, pp. 287-290.

[5] Khayatzadeh R., Auroux V., Fernandez A., Llopis O., COEO phase locking and performance optimisation. Proc. of the IEEE EFTF-IFCS 2017 joint conference, Besançon 2017.

[6] Maleki, L., 2017, June. Optoelectronic oscillators for microwave and mmwave generation. In Radar Symposium (IRS), 2017 18th International (pp. 1-5). IEEE.

[7] Matsko, A.B., Eliyahu, D., Koonath, P., Seidel, D. and Maleki, L., 2009. Theory of coupled optoelectronic microwave oscillator I: expectation values. JOSA B, 26(5), pp.1023-1031.

[8] Matsko, A.B., Eliyahu, D. and Maleki, L., 2013. Theory of coupled optoelectronic microwave oscillator II: phase noise. JOSA B, 30(12), pp.3316-3323.

[9]Agrawal, G. P., Olsson, N. A. (1989). Self-phase modulation and spectral broadening of optical pulses in semiconductor laser amplifiers. IEEE Journal of Quantum Electronics, 25(11), 2297-2306.

[10] Ly, A., Bailly, G., Fernandez, A., and Llopis, O. (2018). Chromatic Dispersion measurement of short optical fibers by sinusoidal phase modulation of a monochromatic light. IEEE Topical Meeting on Microwave Photonics (MWP 2018), Toulouse, Oct. 2018.

[12] Liao, R., Tang, M., Fu, S. and Liu, D. (2018). Distributed Measurement of Polarization Mode Coupling in Polarization Maintaining Fibers using Microwave Photonic Filter Technique. Journal of Lightwave Technology, vol 36, n 19 (2018), pp. 4543-4548. 